\title{
Radiation Effects on Marangoni Boundary Layer Flow in Micropolar Fluid with Suction/Injection
}

\author{
${ }^{1}$ Norfarahanim Mohd Ariffin, ${ }^{2}$ Norihan Md Arifin, ${ }^{3}$ Ahmad Nazri Mohamad Som \\ ${ }^{3}$ Yong Faezah Rahim and ${ }^{1}$ Norfifah Bachok \\ ${ }^{1}$ Department of Mathematics, Faculty of Science, \\ ${ }^{2}$ Institute for Mathematical Research, \\ ${ }^{3}$ Centre of Foundation Studies for Agriculture Science, Universiti Putra Malaysia, \\ 43400 UPM Serdang, Selangor, Malaysia \\ nazrims@upm.edu.my
}

\begin{abstract}
The influence of radiation on the Marangoni boundary layer flow in micropolar fluid with suction/injection is studied in this study. It is the extension of the previous study by Saleh et al. where the interface temperature is assumed to be a quadratic function of the distance $\mathrm{x}$ along the interface. A set of nonlinear ordinary differential equations are obtained by using the technique of similarity transformations, transformed from the general governing partial differential equations and solved numerically by using the shooting method. The solutions of each profiles is presented in the form of tables and figures along with the results of the velocity, microrotation gradient and heat transfer at the surface. The problem is considered for two different values of $\mathrm{n}$ which is $\mathrm{n}=0$ and $\mathrm{n}=0.5$ where it is represented the strong and the weak concentration, respectively with different values of radiation parameter along with the suction/injection parameter.
\end{abstract}

Key words: Radiation effect, Marangoni convection, boundary layer, micropolar fluid, shooting method, radiation parameter

\section{INTRODUCTION}

The Marangoni effect is a phenomenon whereby liquids flow from the area that having low surface tension to the higher surface tension area either along a gas-liquid or liquid-liquid interface. Basically, Marangoni flows are induced by surface tension gradients at the interface of immiscible fluids and the surface tension gradients that are responsible for the Marangoni convection can be due to gradients of temperature and/or concentration (Golia and Viviani, 1986). Convection or also can be referred as convective heat transfer is the transfer of heat from one place to another by the movements of groups of molecules within fluids and rheids. The name of Marangoni was given after an Italian physicist in the 19th century. The study of Marangoni convection has attracted much attention in recent years due to the vast applications in industries. Some of its applications are on the space experimentation under microgravity conditions, semiconductor processing in the field of crystal growth and in art works of dyeing on the ground (Kuroda, 2000).
The technique of dyeing can also be used in the field of science educations, practically for demonstrating one of the space experimentation and can be displayed in thelclassroom. Marangoni became an important part in microgravity science and space craft due to its possibility of material processing in space craft where the gravity force is very small in comparison with the thermo-capillary, (Lian-Cun et al., 2004; Chen, 2007). Marangoni convection can be a main factor of the fluid movements when the gravity is absence.

The original work in this field was first addressed by Napolitano (1979) where he studied the Marangoni boundary layers which can be formed along the interface of two immiscible fluids in surface driven flow. The behaviors, structures and the properties of Marangoni boundary layers were studied by Napolitano and Golia on which they considered the coupled plane case, thermally and kinematically. Following after, numerous of studied on Marangoni have been widely investigated with many different factors. For such Christopher and Wang (2001) investigate the effect of Prandlt number on Marangoni

Corresponding Author: Ahmad Nazri Mohamad Som, Centre of Foundation Studies for Agriculture Science, Universiti Putra Malaysia, 43400 UPM Serdang, Selangor, Malaysia, nazrims@upm.edu.my 
convection overla flat surface. Zhang and Zheng (2012a, b) analyzed the effect of uniform magnetic field, heat generation and first-order chemical reaction on the flow, heat and mass transfer of Marangoni convection flow. The mixed convection boundary layer generated when there are buoyancy effects due to gravity and external pressure gradients effect along with the Marangoni effect. This case of studied was done by many researchers such as Chamkha et al. (2006) and Zhang and Zheng (2014). Generally, the Marangoni mixed convection parameter has a substantial effect on the flow and heat transfer of the fluid. Pop et al. (2001) studied the case of forced convection boundary layer which is done by many researchers before such as Napolitano and Golia and Christopher and Wang $(2001 \mathrm{a}, \mathrm{b})$ were Pop et al. (2001) investigated the relationship between thermal and solutal concentration. While the case of free convection was done by Golia and Viviani (1985) and Napolitano et al. (1992).

The theory of microfluids was first introduced by Eringen $(1964,1966)$. It is fluids that exhibit a certain microscopic effects arising from the local structure and micro-motions of the fluid elements. These fluids are influences by the spin inertia and can support stress moments and body moments. Micropolar fluids are a subclass of these fluids which exhibit the micro-rotational effects and micro-rotational inertia and it is consist of randomly oriented molecules. These micropolar fluids, however, can support couple stress and body stress only. It is may represent fluids consisting of bar-like or sphere-like elements, physically. Ariman et al. (1973) discussed special features of micropolar fluids in the reviewed paper of the subject and application of micropolar fluid mechanics. These fluids have potential importance in industrial applications which have motivate the study in this area. The prolongation of the main equations for Newtonian fluids, so that, more complex fluids such as particle suspensions, liquid crystals, animal blood, lubrication and turbulent shear flow can be described by this theory is the gist of the theory of the micropolar fluids. Through this theory, a transport equation $\backslash$ which represents the principle of conservation of local angular momentum must be added to the usual transport equations for the conservation of mass and momentum and additional local constitutive parameters are also introduced in practice.

The problems of micropolar fluids have been studied numerously through diverse aspect, for example, by Eringen (1966); Nath (1975); Nazar et al. (2004) etc. Peddieson (1972) investigated the problems of axisymmetric stagnation-point flow of micropolar fluids over a flat plate. It is shown that the model is capable of predicting results which exhibit some characteristics found in the turbulent wall shear layers. Ahmadi (1976) studied the theory of micropolar fluid and its applications to the dynamic of low concentration suspension flows. The stagnation flows of micropolar fluids with a strong and weak concentration where two different boundary conditions for spin were considered was analyzed by Guram and Smith (1980).

Damseh et al. (2009) investigated the effect of heat generation and first-order chemical reaction on micropolar fluids flows over a uniformly stretched permeable surface. It is shown that both heat generation and first-order chemical reaction enhanced the respective heat and mass transfer coefficients and decreased the friction coefficient. Ishak (2010) studied the effects of radiation on the thermal boundary layer flow induced by linearly stretching sheet immersed in an incompressible micropolar fluid with constant surface temperature. The result shows that the existence of radiation decreased the heat transfer rate at the surface.

All substances continuously emit electromagnetic radiation by benefaction of the molecular and atomic agitation connecting with the internal energy of the material. The radiative effects have important applications in physics and engineering particularly in space technology and high operating temperature processes. The basic different between radiation and the conduction and convection, make the radiation becomes an important energy-exchange mechanism at high-absolute-temperature levels. Radiative transfer need no medium present between 2 locations for radiant interchange to occur, contrast with the conduction and convection. Thus when there is no medium, radiation becomes the only significant mode of heat transfer. Technology of solar energy utilization, reject waste heat from a power plant operating in space, wavelength region that give mankind heat, light, photosynthesis and all the attendant benefits are some of the factors why radiation is important (Siegel, 1981). The radiation-convection interaction problems are found in consideration of the cooling high temperature components, furnace design where the heat transfer from surfaces occurs by parallel radiation and convection, convection cells and their effect on radiation from stars, the interaction of incident solar radiation with the Earth's surface to produce complex free convection patterns and thus, to complicate the art of weather forecasting and marine environment studies for predicting free convection patterns in the oceans and lake. 
Extensive study regarding radiation has been investigated by numerous researchers due to its wide applications in industries. Cess (1961) scrutinizes the effect of radiation heat transfer upon the forced-convection Nusselt number and the result denoted between laminar and turbulent flow. It was state that the radiation can be significant for laminar flow, particularly in low density applications for which Reynold number may be quite low but it is almost insignificant for turbulent forced convection on a flat surface. Ishak (2010) analyzed the effect of radiation on thermal boundary layer flow over a stretching sheet in a micropolar fluid, Hamid et al. (2011) examined the radiation effect on Marangoni boundary layer flow past a flat plate in nanofluid, Mat et al. (2013) studied the effect of radiation on Marangoni convection boundary layer over a permeable surface and Lin et al. (2014) studied the effects of radiation parameter on Marangoni convection flow and heat transfer in pseudo-plastic non-Newtonian nanofluids with variable thermal conductivity. All found that the radiation parameter tend to reduce the heat transfer rate at the surface. Reddya et al. (2015) analyzed the influence of chemical reaction, radiation and rotation on MHD nanofluid flow past a permeable flat plat in porous medium.

The results indicate that the radiation and magnetic field parameters have the tendency to diminish the friction factor. The effect of magnetic field, thermal radiation through permeable surface on Marangoni boundary layer flow past a surface embedded in a porous medium saturated by a water based nanofluid containing two different types of nanoparticles was investigated by Aly and Ebaid (2016). It shown that the temperature profiles increase with the radiation and it is generally, state that the radiation can be used to control the thermal boundary layers quite effectively.

An approximate solution to the uniform suction has been given by Ariel (1994). The significance of suction or injection on the boundary layer control in the field of aerodynamics and space science is well recognized by Singh (1984). In general, suction tends to increase the skin friction and heat transfer coefficients whereas injection act in the opposite manner by Al-Sanea (2004). Recently, the studied with the consideration of suction or injection has been widely investigated by many researchers. Al-Mudhaf and Chamkha (2005) examined the effect of heat generation or absorption on the MHD thermosolutal Marangoni convection over a flat surface and they stated that the imposition of suction has the tendency to decrease the fluid velocity and the hydrodynamic boundary layers but the injection shows the opposite result. Attia (2008) considered the effect of uniform suction or blowing on the steady laminar flow of an incompressible non-Newtonian micropolar at two-dimensional stagnation point with heat transfer. While Afify (2009) considered the effect of thermal diffusion and diffusion thermo on free convective heat and mass transfer over stretching surface considering suction or injection. Suction or injection gave a physicaleffect on the local Nusselt number. Mat et al. (2013) studied the effect of suction/injection on the Marangoni convection boundary layer with radiation. It is shown that the presence of suction tend to decrease the surface temperature gradient whereas the injection shows the opposite effect.

The aim of this stusy is to examine the effect of radiation on the Marangoni boundary layer flow in micropolar fluid with the presence of suction/injection, the extension of the previous study by Saleh et al. (2014). The interface temperature assumes to be in quadratic function of the distance $\mathrm{x}$ along the interface. The general governing partial differential equations are transformed into a set of nonlinear ordinary differential equations by using the similarity transformation technique and solve numerically by using the shooting method. Two different values of boundary condition for microrotation $n$ which each of it represented strong and weak concentration of elements is take into account along with the other considered parameters.

\section{MATERIALS AND METHODS}

Mathematical formulations: The steady laminar Marangoni boundary layer of a micropolar fluid is considered and the interface temperature is assumed to be a quadratic function of the distance $\mathrm{x}$ along the interface with a radiation and suction/injection effects. The basic governing equations of the propose problem based on the balance laws of mass, momentum, microrotation and energy which can be written in dimensional form as:

$$
\begin{gathered}
\frac{\partial u}{\partial x}+\frac{\partial v}{\partial y}=0 \\
u \frac{\partial u}{\partial x}+v \frac{\partial v}{\partial y}=\left(\frac{\mu+\kappa}{\rho}\right) \frac{\partial^{2} u}{\partial y^{2}}+\frac{\kappa}{\rho} \frac{\partial N}{\partial y} \\
\rho j\left(u \frac{\partial N}{\partial x}+v \frac{\partial N}{\partial y}\right)=\gamma \frac{\partial^{2} N}{\partial y^{2}}-\kappa\left(2 N+\frac{\partial u}{\partial y}\right) \\
u \frac{\partial T}{\partial x}+v \frac{\partial T}{\partial y}=\alpha \frac{\partial^{2} T}{\partial y^{2}}-\frac{1}{\rho C_{p}} \frac{\partial q_{r}}{\partial y}
\end{gathered}
$$

subject to the boundary conditions: 


$$
\begin{gathered}
v(\mathrm{x}, 0)=\mathrm{f}_{0}, \mu\left(\frac{\partial \mathrm{u}}{\partial \mathrm{y}}\right)=-\left(\frac{\partial \sigma}{\partial \mathrm{x}}\right)=\sigma_{0} \gamma_{\mathrm{T}}\left(\frac{\partial \mathrm{T}}{\partial \mathrm{x}}\right), \mathrm{N}= \\
-\mathrm{n} \frac{\partial \mathrm{u}}{\partial \mathrm{y}}, \mathrm{T}(\mathrm{x}, 0)=\mathrm{T}_{\infty}+\mathrm{T}_{0} \mathrm{X}^{2}, \mathrm{X}=\frac{\mathrm{x}}{\mathrm{L}}, \mathrm{u}(\mathrm{x}, \infty)= \\
0, \mathrm{~N}(\mathrm{x}, \infty)=0, \mathrm{~T}(\mathrm{x}, \infty)=\mathrm{T}_{\infty}
\end{gathered}
$$

The surface tension is conclude to depend on the temperature linearly:

$$
\sigma=\sigma_{0}\left[1-\gamma_{\mathrm{T}}\left(\mathrm{T}-\mathrm{T}_{\infty}\right)\right]
$$

and $\gamma_{\mathrm{T}}$ is indicate as the temperature coefficient of the surface tension:

$$
\gamma_{\mathrm{T}}=-\frac{1}{\sigma_{0}}\left(\frac{\partial \sigma}{\partial \mathrm{T}}\right)
$$

where $\mathrm{u}$ and $\mathrm{v}$ are the velocity components along the $x$ and $y$ axes. $\mathrm{N}, \mathrm{T}, \mathrm{T}_{0}, \mathrm{q}_{\mathrm{r}}$ and $\mathrm{L}$ represent the microrotation, fluid temperature, positive or negative dimensional constant, radiative heat flux and reference length, respectively. We have denoted that $\mu, \kappa, \rho, j, \gamma, \alpha, \rho C_{p}$ and $f_{0}$ as the dynamic viscosity, vortex viscosity, fluid density, spin gradient viscosity, thermal, thermal diffusivity, heat capacity and suction/injection parameter, respectively. While $\mathrm{n}$ is a constant that varies in the range of $0 \leq n \leq 1$. The strong concentration is denoted by $n=0$ as stated by Guram and Smith (1980) and N = 0 near the wall represents concentrated particle flows in which the microelements close to the wall surface are unable to rotate. While $n=0.5$ represented the weak concentration which indicates the vanishing of the anti-symmetric part of the stress tensor and as prompted by Ahmadi (1976), $\mathrm{n}=1$ represented the modeling of turbulent boundary layer flows. By succeeding the research of many recent researchers, we assuming that:

$$
\gamma=\left(\mu+\frac{\kappa}{2}\right) j=\mu\left(1+\frac{\kappa}{2}\right) j
$$

where, $\mathrm{K}=\kappa / \mu$ is the micropolar parameter (Saleh et al., 2014). The similarity variables are defined as:

$$
\begin{array}{rc}
\psi(\mathrm{x}, \mathrm{y})=v \operatorname{Xf}(\eta) \quad \mathrm{N}(\mathrm{x}, \mathrm{y})=\left(\frac{\mathrm{y}}{\mathrm{L}^{2}}\right) \operatorname{Xg}(\eta) \\
\mathrm{T}(\mathrm{x}, \mathrm{y})=\mathrm{T}_{\infty}+\mathrm{T}_{0} \mathrm{X}^{2} \theta(\eta) \quad \eta=\frac{\mathrm{y}}{\mathrm{L}}
\end{array}
$$

where, $\psi$ is the stream function which defined as $\mathrm{u}=$ $\partial \psi / \partial y$ and $v=-\partial \psi / \partial y$. For radiation by using the Rosseland approximation (Brewster, 1992) the radiative heat flux can be simplified as:

$$
\mathrm{q}_{\mathrm{r}}=-\frac{4 \sigma^{*}}{3 \mathrm{k}^{*}} \frac{\partial \mathrm{T}^{4}}{\partial \mathrm{y}}
$$

Where:

$\boldsymbol{\sigma}^{*}=$ The Stefan-Boltzmann constant

$\mathrm{k}^{*}=$ The mean absorption coefficients

It is assumed that the temperature differences within the flow such the term $\mathrm{T}^{4}$ can be expressed as a linear function of temperature. This can be accomplished by expanding $T^{4}$ in a Taylor's series about $T_{\infty}$ and neglecting the higher-order terms, thus:

$$
\mathrm{T}^{4} \approx 4 \mathrm{~T}_{\infty}^{3} \mathrm{~T}-3 \mathrm{~T}_{\infty}^{4}
$$

Using Eq. 9 and 10, Eq. 4 reduces to,

$$
\mathrm{u} \frac{\partial \mathrm{T}}{\partial \mathrm{x}}+v \frac{\partial \mathrm{T}}{\partial \mathrm{y}}=\alpha(1+\mathrm{Nr}) \frac{\partial^{2} \mathrm{~T}}{\partial \mathrm{y}^{2}}
$$

Substituting Eq. (8) into Eq. 1-3 and Eq. 1, we get the following ordinary differential equations:

$$
\begin{gathered}
(1+\mathrm{K}) \mathrm{f}^{\prime \prime \prime}+\mathrm{ff}^{\prime \prime}-\mathrm{f}^{12}+\mathrm{Kg} \mathrm{g}^{\prime}=0 \\
(1+\mathrm{K} / 2) \mathrm{g}^{\prime \prime}+\mathrm{fg} \mathrm{g}^{\prime \prime}-\mathrm{f}^{\prime} \mathrm{g}-\mathrm{K}\left(2 \mathrm{~g}+\mathrm{f}^{\prime \prime}\right)=0 \\
\frac{1}{\mathrm{Pr}}(1+\mathrm{Nr}) \theta^{\prime \prime}+\mathrm{f}^{\prime}-2 \mathrm{f}^{\prime} \theta=0
\end{gathered}
$$

Subject to the boundary conditions:

$$
\begin{gathered}
f(0)=S, f^{\prime \prime}(0)=-2, g(0)=-n f^{\prime \prime}(0)=2 n, \theta(0)=1(15) \\
f^{\prime}(\infty)=0, g(\infty)=0, \theta(\infty)=0
\end{gathered}
$$

Where:

Prime $=$ The differentiation respect to $\eta$

$\mathrm{K}=$ The material parameter

$\mathrm{P}_{\mathrm{r}} \quad=\quad$ The Prandtl number

$\mathrm{N}_{\mathrm{r}}=$ Represent radiation parameter

$\mathrm{S}=$ The suction/injection parameter and the reference length, $L$ is chosen as:

$$
\mathrm{L}=-\frac{\mu \nu}{\sigma_{0} \gamma_{\mathrm{T}} \mathrm{T}_{0}}
$$

Increasing the temperature in general will decrease the surface tension, $\sigma$ and the temperature gradient, $\gamma_{\mathrm{T}}$ is positive. Thus, the reference Length $\mathrm{L}$ chosen, according to the Eq. 16 is positive only if $T_{0}$ is negative (Christopher and Wang, 2001a, b). 


\section{RESULTS AND DISCUSSION}

The nonlinear ordinary differential Eq. 12-14 subject to boundary conditions Eq. 15 were solve numerically using the shooting method. The values of surface velocity $f(0)$, gradient of the microrotation $g^{\prime}(0)$ and the heat transfer at the surface, $-\theta^{\prime}(0)$ as well as the velocity $f(0)$, angular velocity or microrotation $g(\eta)$ and temperature $\theta(\eta)$ profiles are obtain for different values of microrotation parameter $\mathrm{n}$, radiation parameter $\mathrm{Nr}$ and suction/injection parameter $\mathrm{S}$ along with the fixed value of material parameter, $\mathrm{K}=1$ and Prandtl number, $\mathrm{Pr}$ $=0.78$. The two values of $n, n=0$ and $n=0.5$ represents the strong and weak concentration of microelements being considered in this study. Values of $\mathrm{f}^{\prime}(0), \mathrm{g}^{\prime}(0)$ and $-\theta^{\prime}(0)$ for different radiation parameter are given in the Table 1 for both case weak and strong concentration. The results without radiation are compared with the previous study by Saleh et al. (2014) and it is found to be in a good agreement. Table 2 represent the results of $f(0)$, $g^{\prime}(0)$ and $-\theta^{\prime}(0)$ for different value of suction/injection parameter, $\mathrm{S}$ with both strong and weak concentration. It is also compared with Saleh et al. (2014) when the suction/injection parameter are neglected and found to be in good agreement. It should be mentioned that $\mathrm{f}(0)$ and $-\theta^{\prime}(0)$ are related to the velocity at the surface and Nusselt number.

Table 1: Values of $f(0), g^{\prime}(0)$ and $-\theta^{\prime}(0)$ for different values of $N r$ when $K=1, \operatorname{Pr}=0.78$ and $S=0$

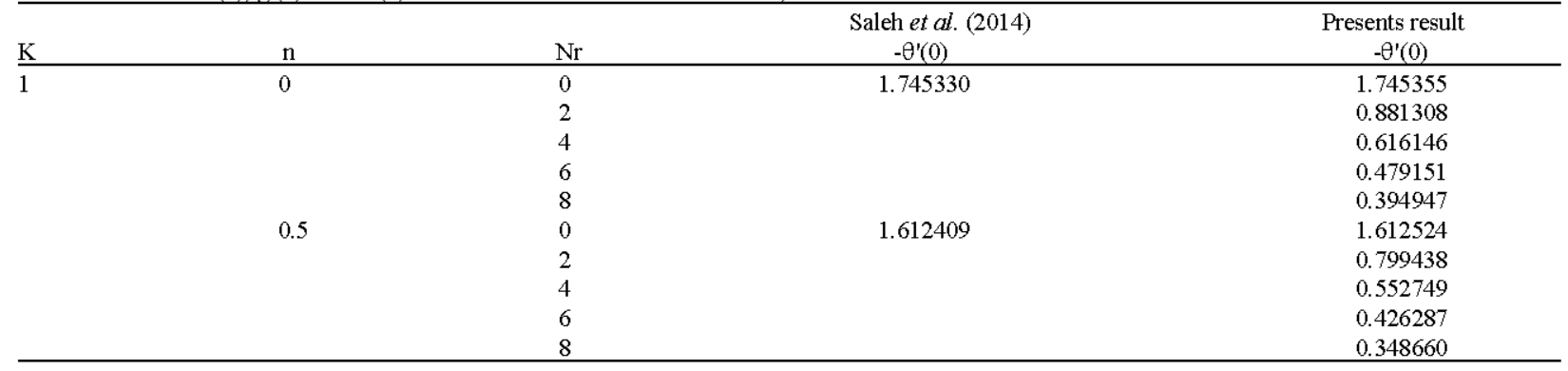

\begin{tabular}{|c|c|c|c|c|c|c|c|c|}
\hline \multirow[b]{2}{*}{$\mathrm{n}$} & \multirow[b]{2}{*}{$\mathrm{Nr}$} & \multirow[b]{2}{*}{$\mathrm{S}$} & \multicolumn{3}{|c|}{ Saleh et al. (2014) } & \multicolumn{3}{|c|}{ Presents results } \\
\hline & & & $f^{\prime}(0)$ & $\mathrm{g}^{\prime}(0)$ & $-\theta^{\prime}(0)$ & $f^{\prime}(0)$ & $\mathrm{g}^{\prime}(0)$ & $-\theta^{\prime}(0)$ \\
\hline \multirow[t]{15}{*}{$\overline{0}$} & 0 & -2 & & & & 2.6900252 & 0.4653475 & 1.3991692 \\
\hline & & -1 & & & & 2.3679452 & 0.5272693 & 1.5492450 \\
\hline & & 0 & 2.029266 & 0.5799422 & 1.745330 & 2.0292952 & 0.5793995 & 1.7453557 \\
\hline & & 1 & & & & 1.6889646 & 0.6049889 & 1.0154202 \\
\hline & & 2 & & & & 1.3740965 & 0.5929933 & 2.3930987 \\
\hline & 2 & -2 & & & & 2.6900247 & 0.4653480 & 0.8662182 \\
\hline & & -1 & & & & 2.3679451 & 0.5272693 & 0.8736422 \\
\hline & & 0 & & & & 2.0292952 & 0.5793995 & 0.8813382 \\
\hline & & 1 & & & & 1.6889646 & 0.6049889 & 0.9042052 \\
\hline & & 2 & & & & 1.3740965 & 0.5929933 & 0.9653458 \\
\hline & 5 & -2 & & & & 2.6900251 & 0.4653475 & 0.5903904 \\
\hline & & -1 & & & & 2.3679452 & 0.5272693 & 0.5652970 \\
\hline & & 0 & & & & 2.0292952 & 0.5793995 & 0.5385467 \\
\hline & & 1 & & & & 1.6889646 & 0.6049889 & 0.5206380 \\
\hline & & 2 & & & & 1.3740965 & 0.5929933 & 0.5287102 \\
\hline \multirow[t]{15}{*}{0.5} & 0 & -2 & & & & 2.5251022 & -0.7920471 & 1.3223592 \\
\hline & & -1 & & & & 2.1799810 & -0.9174391 & 1.4469612 \\
\hline & & 0 & 1.816998 & -1.100499 & 1.612409 & 1.8171205 & -1.1006424 & 1.6125246 \\
\hline & & 1 & & & & 1.4561642 & -1.3734714 & 1.8571180 \\
\hline & & 2 & & & & 1.1347284 & -1.7625362 & 2.2329464 \\
\hline & 2 & -2 & & & & 2.5251022 & -0.7920471 & 0.8167755 \\
\hline & & -1 & & & & 2.1799810 & -0.9174391 & 0.8098180 \\
\hline & & 0 & & & & 1.8171205 & -1.1006424 & 0.7994386 \\
\hline & & 1 & & & & 1.4561642 & -1.3734714 & 0.8065357 \\
\hline & & 2 & & & & 1.1347284 & -1.7625362 & 0.8660547 \\
\hline & 5 & -2 & & & & 2.5251022 & -0.7920471 & 0.5543745 \\
\hline & & -1 & & & & 2.1799810 & -0.9174391 & 0.5195927 \\
\hline & & 0 & & & & 1.8171205 & -1.1006424 & 0.4809026 \\
\hline & & 1 & & & & 1.4561642 & -1.3734714 & 0.4548230 \\
\hline & & 2 & & & & 1.1347284 & -1.7625362 & 0.4636352 \\
\hline
\end{tabular}




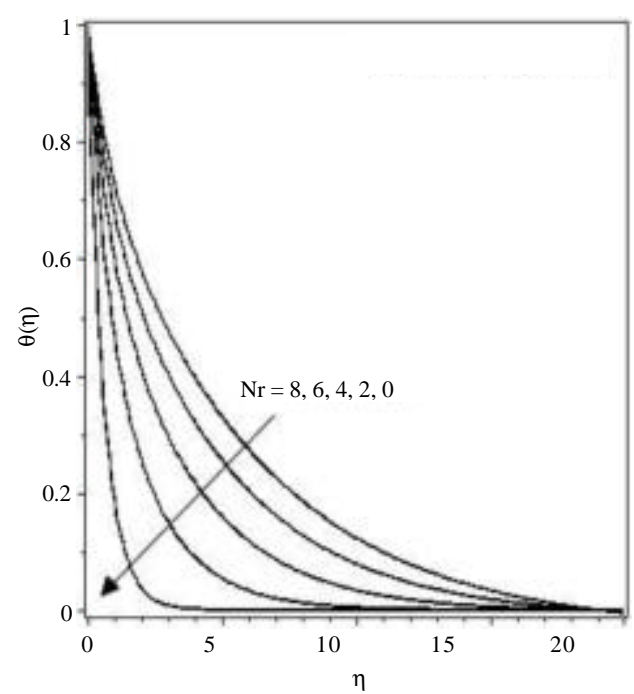

Fig. 1: The effects of $\mathrm{Nr}$ on the $\theta(\eta)$ when $\mathrm{K}=1, \mathrm{Pr}=$ $0.78, \mathrm{~S}=0$ and $\mathrm{n}=0$

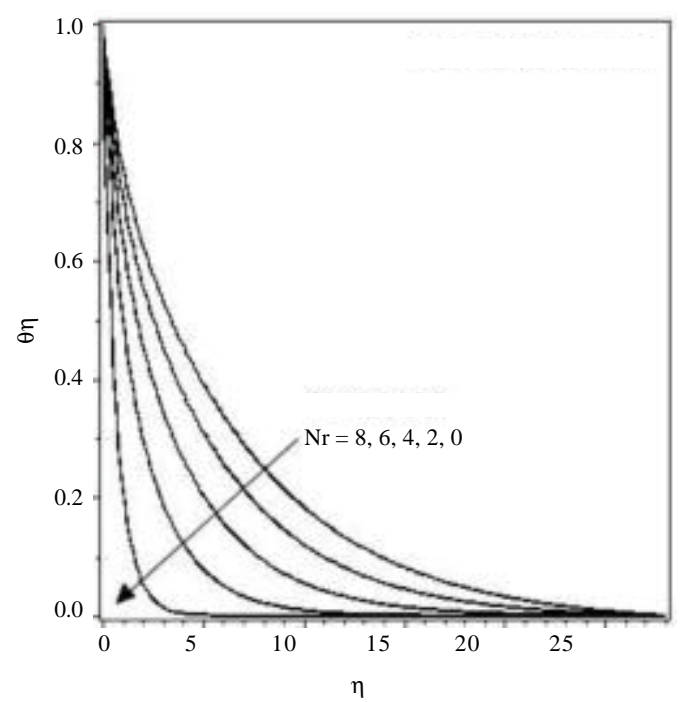

Fig. 2: The effects of $\mathrm{Nr}$ on the $\theta(\eta)$ when $\mathrm{K}=1, \mathrm{Pr}=$ $0.78, \mathrm{~S}=0$ and $\mathrm{n}=0.5$

Figure 1 and 2 show the effect of radiation parameter, $\mathrm{Nr}$ on the temperature profile, $\theta(\eta)$ for both strong and weak concentration of miroelements, $\mathrm{n}=0$ and $\mathrm{n}=0.5$. We noted that radiation parameter has no influence on the flow field which is clear from Eq. 12-14. It is can be clearly seen that the temperature profile increase as the radiation increase. This is due to the releasing of more heat to the flow as the radiation increase, since, the radiation parameter is the measure of relative importance of the thermal radiation transfer to the conduction of heat. It means the larger the value of radiation parameter, the more

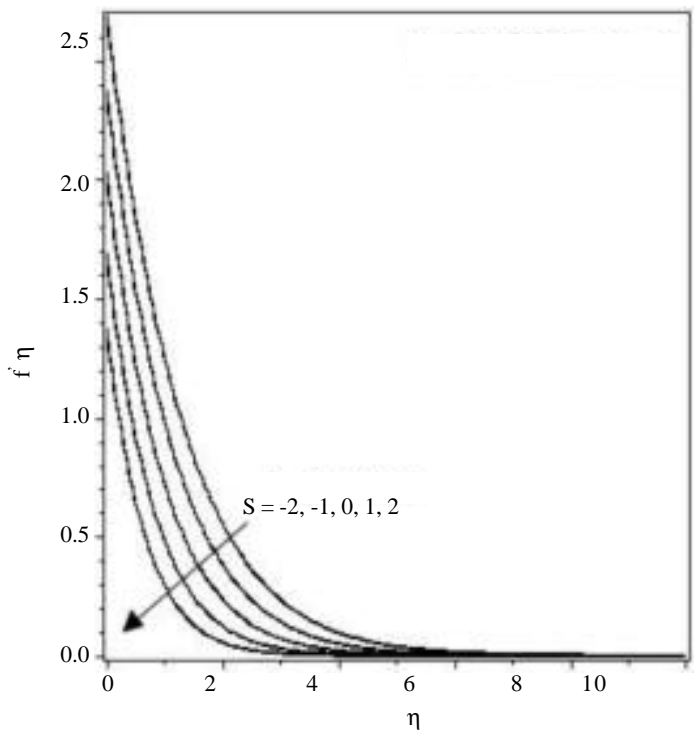

Fig. 3: The effects of $S$ on the $f^{\prime}(\eta)$ when $K=1, \operatorname{Pr}=0.78$, $\mathrm{Nr}=0$ and $\mathrm{n}=0$

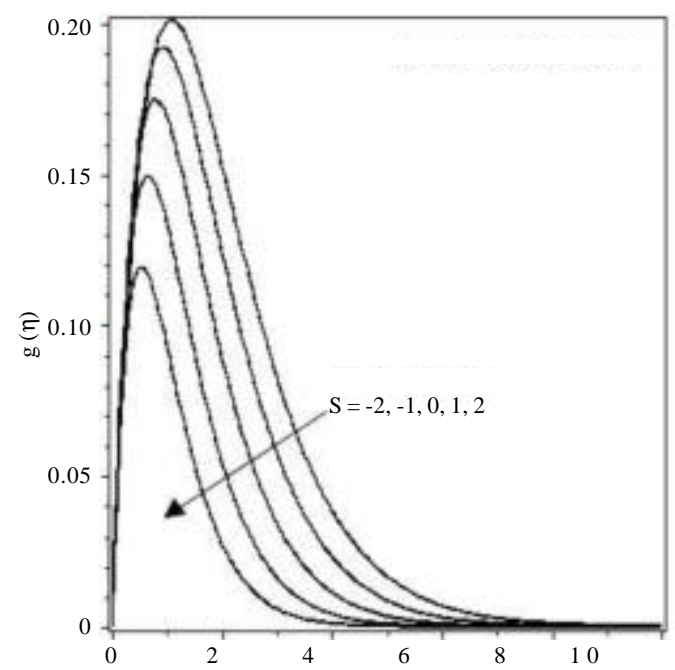

Fig. 4: The effects of $\mathrm{S}$ on the $\mathrm{g}(\eta)$ when $\mathrm{K}=1, \operatorname{Pr}=0.78$, $\mathrm{Nr}=0$ and $\mathrm{n}=0$

radiative heat energy being transfer into the system and causing the rise in the temperature profile which then enhances the momentum and thermal boundary layer thickness. Thus, it can be conclude that radiation can be used to control the thermal boundary layers quite effectively. Furthermore, the strong concentration gives a higher value than the weak concentration for the effect of radiation on temperature profiles.

Figure 3-8 present the results for the effect of suction/injection parameter, $S$ on the velocity $f(\eta)$, angular velocity $g(\eta)$ and temperature profiles $\theta(\eta)$ for 


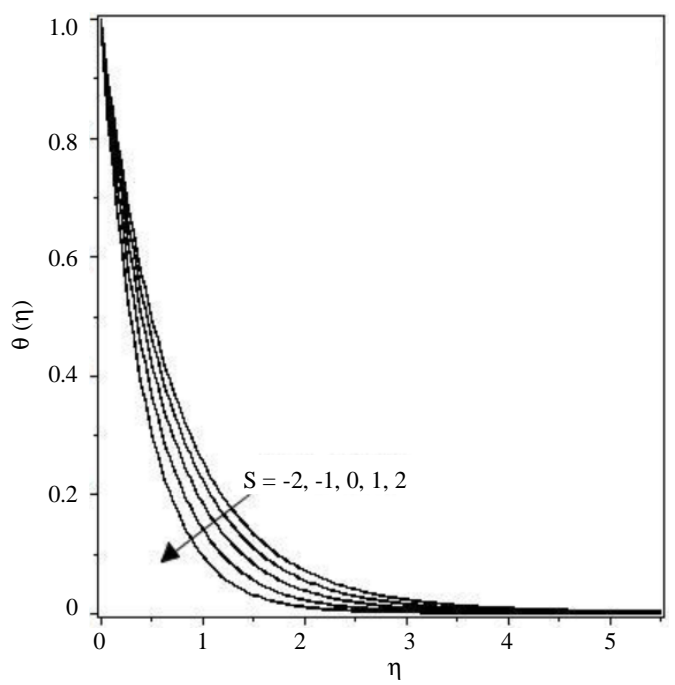

Fig. 5: The effects of $S$ on the $\theta(\eta)$ when $K=1, \operatorname{Pr}=0.78$, $\mathrm{Nr}=0$ and $\mathrm{n}=0$

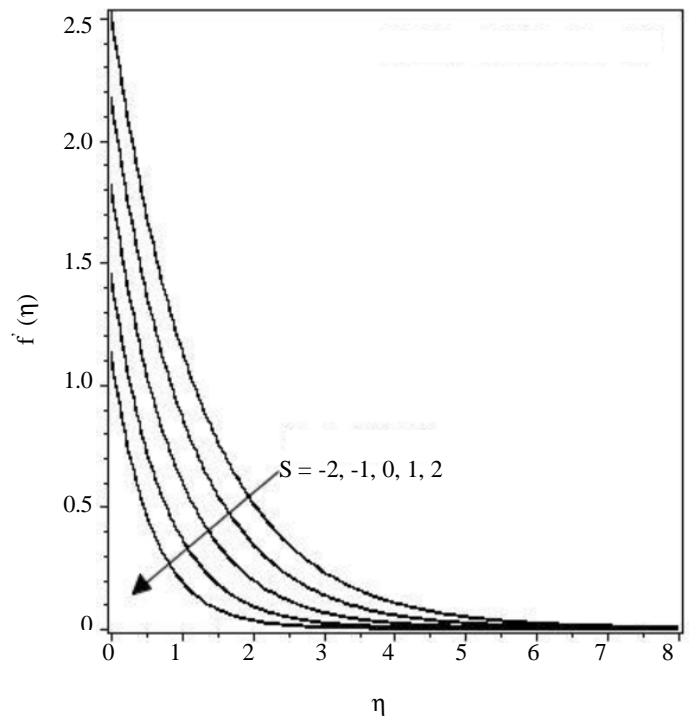

Fig. 6: The effects of $S$ on the $f(\eta)$ when $K=1, \operatorname{Pr}=0.78$, $\mathrm{Nr}=0$ and $\mathrm{n}=0.5$

both case of strong and weak concentrations of microelements. Figure 3-5 are the results for the strong concentration while Fig. 4-8 represents the results for the weak concentration. The imposition of suction $(\mathrm{S}>0)$ at the surface has the tendency to reduce the velocity, angular velocity and the temperature of the flow for both strong and weak concentration. As the fluid moves nearer to the surface, it tends to decrease the velocity and thermal boundary layer thickness. Thus, the velocity and angular velocity along with the temperature profiles decrease with the inclusion of suction. The opposite

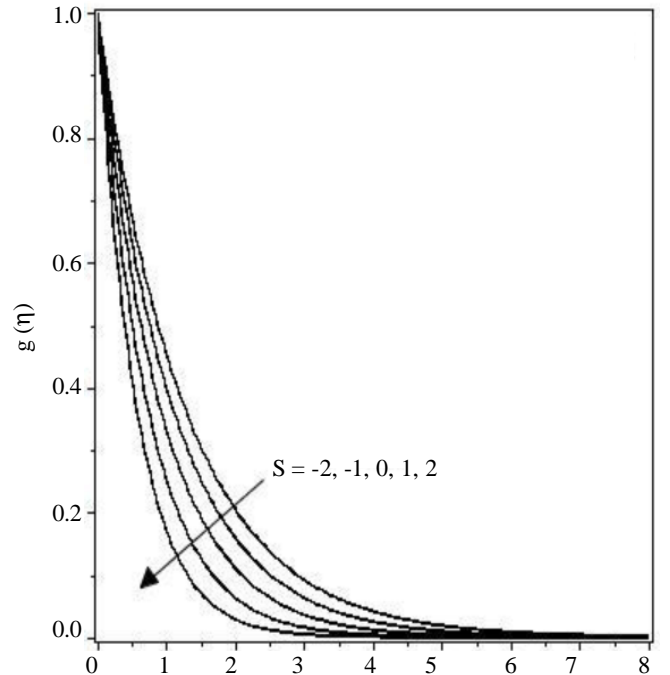

Fig. 7: The effects of $S$ on the $g(\eta)$ when $K=1, \operatorname{Pr}=0.78$, $\mathrm{Nr}=0$ and $\mathrm{n}=0.5$

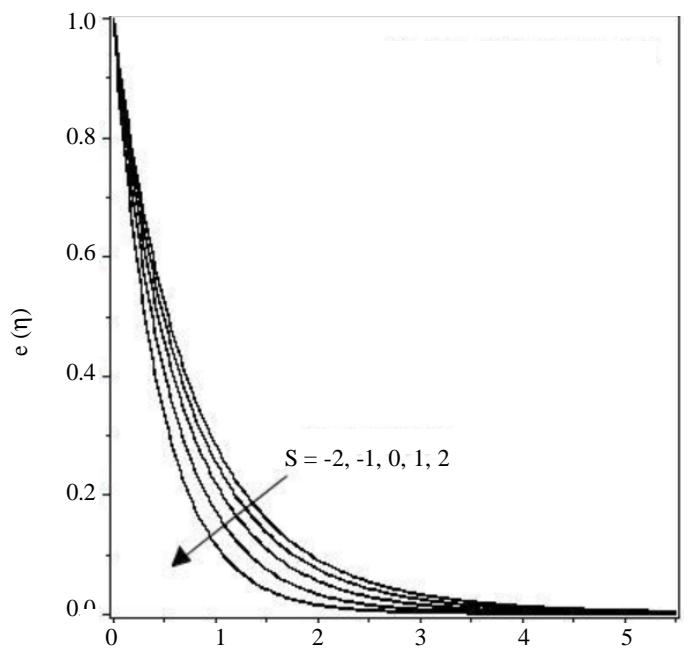

(ๆ)

Fig. 8: The effects of $S$ on the $\theta(\eta)$ when $K=1, \operatorname{Pr}=0.78$, $\mathrm{Nr}=0$ and $\mathrm{n}=0.5$

results are found in the case of injection $(\mathrm{S}<0)$ parameter for velocity, angular velocity and temperature profiles in both case of concentration. It is clearly shown from Fig. 3-8 the imposition of injection will increase the velocity, angular velocity and temperature profiles.

Figure 9 shows the variations of the surface temperature gradient $-\theta^{\prime}(0)$ with radiation for both cases of strong and weak concentrations. It is shown that the surface temperature decrease as the radiation parameter increase. This observation is in agreement with the results presented in Table 1 as the value of $-\theta^{\prime}(0)$ decrease 


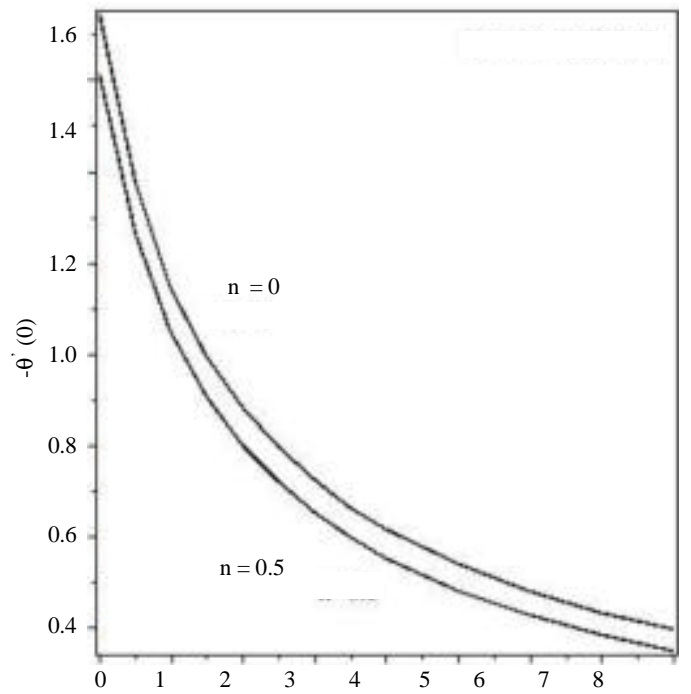

Fig. 9: Variation of $-\theta^{\prime}(0)$ with ${ }^{\mathrm{N}} \mathrm{Nr}$ for $\mathrm{n}=0$ and $\mathrm{n}=0.5$ when $\mathrm{K}=1, \operatorname{Pr}=0.78$ and $\mathrm{S}=0$

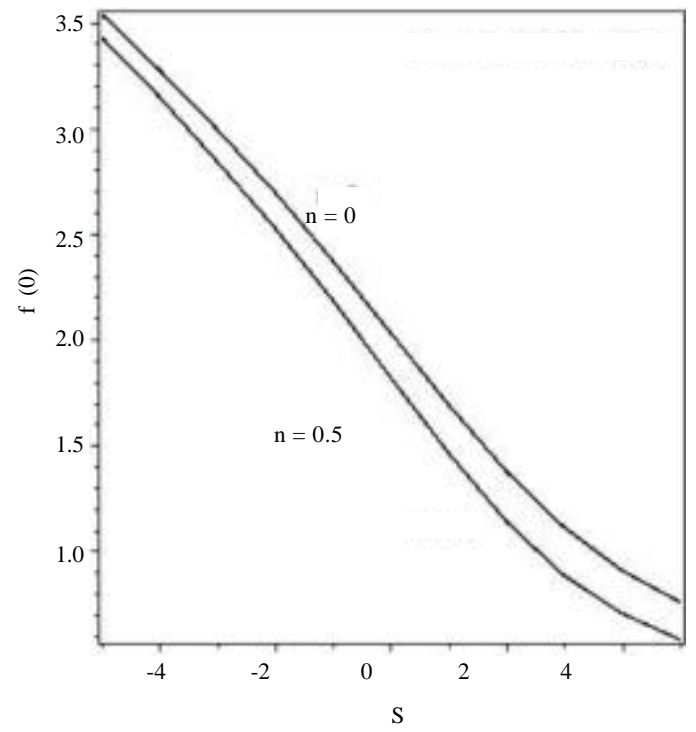

Fig. 10: Variation of $-\theta^{\prime}(0)$ with $S$ for $n=0$ and $n=0.5$ when $\mathrm{K}=1$ and $\mathrm{Pr}=0.78$

when $\mathrm{Nr}$ increases. Thus, the presence of radiation decreases the heat transfer rate at the surface. This is due to the effect of radiation which to reduce the energy transfer rate to the fluid, thereby decreasing the fluid temperature. As the value of heat transfer at the surface $-\theta^{\prime}(0)$ decrease, consequently, the value of Nusselt number also decreases.

Figure 10-12 display the variations values of surface velocity $f^{\prime}(0)$, gradient of the microrotation $g^{\prime}(0)$ and heat transfer rate at the surface $-\theta^{\prime}(0)$ with suction/injection

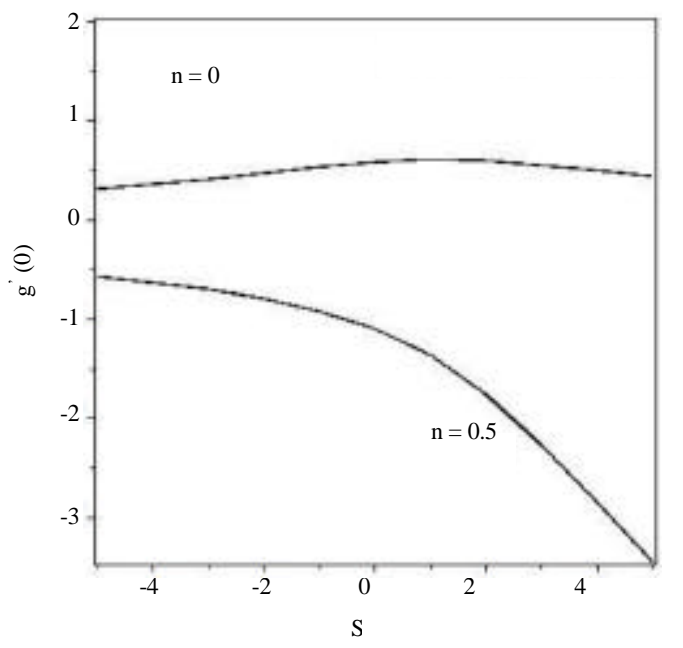

Fig. 11: Variation of $g^{\prime}(0)$ with $S$ for $n=0$ and $n=0.5$ when $\mathrm{K}=1$ and $\mathrm{Pr}=0.78$

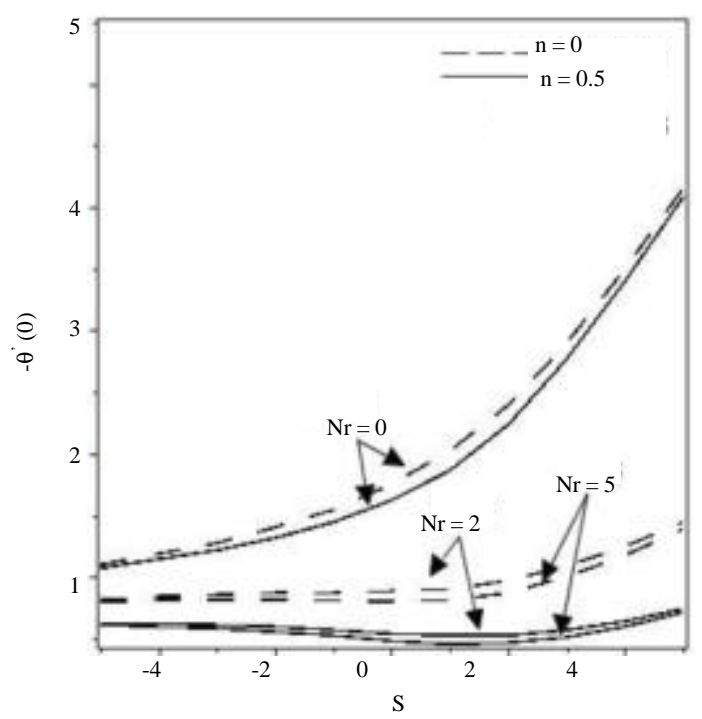

Fig. 12: Variation of $\mathrm{g}^{\prime}(0)$ with $\mathrm{S}$ for $\mathrm{n}=0$ and $\mathrm{n}=0.5$ when $\mathrm{K}=1$ and $\mathrm{Pr}=0.78$

parameter, $\mathrm{S}$ for strong and weak concentrations, $\mathrm{n}=0$ and $\mathrm{n}=0.5$, respectively. It is shown that the implementation of suction $(\mathrm{S}>0)$ tends to decrease the velocity and the microrotation gradient at the surface but increase the temperature at the surface along with the local Nusselt number with or without radiation. The different is the values obtained are slightly lower under the presence of radiation compared with values attained when the radiation is neglected, for all the profiles, Table 2. As the fluid been dragged near to the surface, it caused the decrement on the velocity at the surface as well as the surface microrotation gradient while the surface temperature gradient indicate an increment 
when the suction are implemented. The opposite results are revealed for the injection $(\mathrm{S}<0)$ parameter as can be seen from Table 2 and Fig. 10-12. The surface velocity, microrotation gradient at the surface tends to increase while and the surface heat transfer rate decrease under the presence of injection parameter.

\section{CONCLUSION}

This study presented the steady laminar Marangoni boundary layer flow in micropolar fluid with the presence of radiation and suction/injection effect. The general governing partial differential equations are transformed into a set of nonlinear ordinary differential equations by using the technique of similarity transformations. The effect of the parameters considered on the flow characteristics has been studied and the solutions are obtained numerically by using the shooting method. It was found that the existence of radiation tends to decrease the surface temperature along with the Nusselt number and the strong concentration gives slightly higher values than the weak concentration in the presence of radiation parameter. The implementation of suction parameter on the flow of the fluid incline to decrease the interface velocity and the microrotation gradient at the surface but opposite result happen for the surface temperature gradient. While the injection parameter shown an opposite result which it tends to increase the interface velocity, mirorotation gradient at the surface and alleviate the surface temperature gradient.

\section{ACKNOWLEDGEMENT}

The researchers wish to acknowledge the financial supports received from Putra Grant from Universiti Putra Malaysia.

\section{REFERENCES}

Afify, A.A., 2009. Similarity solution in MHD: Effects of thermal diffusion and diffusion thermo on free convective heat and mass transfer over a stretching surface considering suction or injection. Commun. Nonlinear Sci. Numer. Simul., 14: 2202-2214.

Ahmadi, G., 1976. Self-similar solution of imcompressible micropolar boundary layer flow over a semi-infinite plate. Intl. J. Eng. Sci., 14: 639-646.

Al-Mudhaf, A. and A.J. Chamkha, 2005. Similarity solutions for MHD thermosolutal Marangoni convection over a flat surface in the presence of heat generation or absorption effects. Heat Mass Transfer, 42: 112-121.
Al-Sanea, S.A., 2004. Mixed convection heat transfer along a continuously moving heated vertical plate with suction or injection. Intl. J. Heat Mass Transfer, 47: 1445-1465.

Aly, E.H. and A. Ebaid, 2016. Exact analysis for the effect of heat transfer on MHD and radiation Marangoni boundary layer nanofluid flow past a surface embedded in a porous medium. J. Mol. Liq., 201 5: 625639.

Ariel, P.D., 1994. Hiemenz flow in hydromagnetics. Acta Mech., 103: 31-43.

Ariman, T., M.A. Turk and N.D. Sylvester, 1973. Microcontinuum fluid mechanics-a review. Intl. J. Eng. Sci., 11: 905-930.

Attia, H.A., 2008. Stagnation point flow and heat transfer of a micropolar fluid with uniform suction or blowing. J. Braz. Soc. Mech. Sci. Eng., 30: 51-55.

Brewster, M.Q., 1992. Thermal Radiative Transfer and Properties. John Wiley \& Sons, Hoboken, New Jersey, USA., ISBN:0-471-53982-1, Pages: 514.

Cess, R.D., 1961. The effect of radiation upon forced-convection heat transfer. Appl. Sci. Res., 10: 430-438.

Chamkha, A.J., I. Pop and H.S. Takhar, 2006. Marangoni mixed convection boundary layer flow. Meccanica, 41: 219-232.

Chen, C.H., 2007. Marangoni effects on forced convection of power-law liquids in a thin film over a stretching surface. Phys. Lett. A., 370: 51-57.

Christopher, D.M. and B. Wang, 2001 a. Prandtl number effects for Marangoni convection over a flat surface. Int1. J. Therm. Sci., 40: 564-570.

Christopher, D.M. and B.X. Wang, 2001b. Similarity simulation for Marangoni convection around a vapor bubble during nucleation and growth. Intl. J. Heat Mass Transfer, 44: 799-810.

Damseh, R.A., M.Q. Al-Odat, A.J. Chamkha and B.A. Shannak, 2009. Combined effect of heat generation or absorption and first-order chemical reaction on micropolar fluid flows over a uniformly stretched permeable surface. Intl. J. Therm. Sci., 48: 1658-1663.

Eringen, A.C., 1964. Simple microfluids. Intl. J. Eng. Sci., 2 : 205-217.

Eringen, A.C., 1966. Theory of micropolar fluids. J. Math. Mech., 16: 1-18.

Golia, C. and A. Viviani, 1985. Marangoni-bouyant boundary layers. L. Aerotecnica Missili Spazio, 65: 29-35.

Golia, C. and A. Viviani, 1986. Non isobaric boundary layers related to Marangoni flows. Meccanica, 21: 200-204. 
Guram, G.S. and A.C. Smith, 1980. Stagnation flows of micropolar fluids with strong and weak interactions. Comput. Math. Appl., 6: 213-233.

Hamid, R.A., N.M. Arifin, R.M. Nazar and I. Pop, 2011. Radiation effects on Marangoni boundary layer flow past a flat plate in nanofluid. Proceedings of the International MultiConference on Engineers and Computer Scientists, March 16-18, 2011, International Association of Engineers, Hong Kong, China, ISBN:978-988-19251-2-1, pp: 1260-1263.

Ishak, A., 2010. Thermal boundary layer flow over a stretching sheet in a micropolar fluid with radiation effect. Meccanica, 45: 367-373.

Kuroda, T., 2000. The Marangoni effect and its artistic application. Form., 15: 203-204.

Lian-Cun, Z., C. Xue-Hui, Z. Xin-Xin and H. Ji-Cheng, 2004. An approximately analytical solution for the Marangoni convection in an In-Ga-Sb system. Chin. Phys. Lett., 21: 1983-1985.

Lin, Y., L. Zheng and X. Zhang, 2014. Radiation effects on Marangoni convection flow and heat transfer in pseudo-plastic non-Newtonian nanofluids with variable thermal conductivity. Intl. J. Heat Mass Transfer, 77: 708-716.

Mat, N.A., N.M. Arifin, R. Nazar, F. Ismail and I. Pop, 2013. Radiation effects on Marangoni convection boundary layer over a permeable surface. Meccanica, 48: 83-89.

Napolitano, L.G., 1979. Marangoni boundary layers. Mater. Sci. Space, 1: 349-358.

Napolitano, L.G., A. Viviani and R. Savino, 1992. Double-diffusive boundary layers along vertical free surfaces. Intl. J. Heat Mass Transfer, 35: 1003-1025.
Nath, G., 1975. Similar solutions for the incompressible laminar boundary layer with pressure gradient in micropolar fluids. Rheol. Acta, 14: 850-857.

Nazar, R., N. Amin, D. Filip and I. Pop, 2004. Stagnation point flow of a micropolar fluid towards a stretching sheet. Intl. J. Non Linear Mech., 39: 1227-1235.

Peddieson Jr, J., 1972. An application of the micropolar fluid model to the calculation of a turbulent shear flow. Intl. J. Eng. Sci., 10: 23-32.

Pop, I., A. Postelnicu and T. Grosan, 2001. Thermosolutal marangoni forced convection boundary layers. Meccanica, 36: 555-571.

Reddya, J.V.R., V. Sugunamma, N. Sandeep and C. Sulochana, 2015. Influence of chemical reaction, radiation and rotation on $\mathrm{MHD}$ nanofluid flow past a permeable flat plate in porous medium. J. Niger. Math. Soc., 35: 48-65.

Saleh, S.H., N.M. Arifin, R. Nazar, F.M. Ali and I. Pop, 2014. Marangoni boundary layer flow in micropolar fluid. Aust. J. Basic Appl. Sci., 8: 12-23.

Siegel, R., 1981. Thermal Radiation Heat Transfer (Series in Thermal and Fluid Engineering). 2nd Edn., Hemisphere Publishing Corporation, Washington, DC., USA., ISBN-13:978-0070573161, Pages: 862.

Singh, A.K., 1984. Stokes problem for a porous vertical plate with heat sinks by finite difference method. Astrophys. Space Sci., 103: 241-248.

Zhang, Y. and L. Zheng, 2012. Analysis of MHD thermosolutal Marangoni convection with the heat generation and a first-order chemical reaction. Chem. Eng. Sci., 69: 449-455.

Zhang, Y. and L. Zheng, 2014. Similarity solutions of Marangoni convection boundary layer flow with gravity and external pressure. Chin. J. Chem. Eng., 22: 365-369. 